\title{
Publisher's Note: Intertwined and vestigial order with ultracold atoms in multiple cavity modes
} [Phys. Rev. A 96, 063828 (2017)]

\author{
Sarang Gopalakrishnan, Yulia E. Shchadilova, and Eugene Demler
}

(Q) (Received 16 August 2018; published 28 August 2018)

DOI: 10.1103/PhysRevA.98.029901

This paper was published online on 19 December 2017 with an omission in the Acknowledgments. On page 8, the last sentence of the Acknowledgments should read as "S.G. acknowledges support from the NSF under Grant No. DMR-1653271." The Acknowledgments have been corrected as of 16 August 2018. The Acknowledgments are incorrect in the printed version of the journal. 